PROCEEDINGS OF THE

AMERICAN MATHEMATICAL SOCIETY

Volume 125, Number 1, January 1997, Pages 41-45

S 0002-9939(97)03556-9

\title{
RATIONALLY EQUIVALENT NILPOTENT GROUPS AND SPACES
}

\author{
JOSEPH ROITBERG
}

(Communicated by Thomas Goodwillie)

\begin{abstract}
Examples are constructed of rationally isomorphic, finitely generated nilpotent groups $L$ and $M$ such that there are no homomorphisms $L \rightarrow M, M \rightarrow L$ inducing rational isomorphisms. Similar examples are constructed of nilpotent, or even simply-connected, finite CW-complexes.
\end{abstract}

\section{INTRODUCTION AND STATEMENT OF RESULTS}

Recall that nilpotent spaces $X$ and $Y$ are rationally equivalent if their rationalizations $X_{(0)}$ and $Y_{(0)}$ are homotopy equivalent; similarly, nilpotent groups $L$ and $M$ are rationally isomorphic if their rationalizations $L_{(0)}$ and $M_{(0)}$ are isomorphic. (See [2] for an exposition of the theory of localization of nilpotent groups and spaces.)

In certain situations in homotopy theory, it is important to know, for given rationally equivalent, finite type nilpotent spaces $X$ and $Y$, whether there actually is a map from, say, $X$ to $Y$ inducing a homotopy equivalence $X_{(0)} \simeq Y_{(0)}$. In the context of phantom map theory, several examples were given in [3], [4], [8] to illustrate that such maps need not exist. A particularly simple example of this type, in which there are no maps between $X$ and $Y$ in either direction inducing a homotopy equivalence on the rationalized spaces, is given by the 1-connected, infinite CW-complexes $X=$ the classifying space $B S^{3}$ and $Y=$ the loop space $\Omega S^{5}$ ([8, section 6$])$.

For this example, we have

$$
X_{(0)} \simeq K(\mathbf{Q}, 4) \simeq Y_{(0)},
$$

but for every prime $\ell$, the $\ell$ - localizations $X_{(\ell)}$ and $Y_{(\ell)}$ are not homotopy equivalent. In contrast, if $X$ and $Y$ are rationally equivalent, 1-connected, finite $\mathrm{CW}$ complexes, then it turns out that $X_{(\ell)} \simeq Y_{(\ell)}$ for almost all $\ell$; similarly, if $L$ and $M$ are rationally isomorphic, finitely generated nilpotent groups, then $L_{(\ell)} \cong M_{(\ell)}$ for almost all $\ell{ }^{1}$

Received by the editors May 5, 1995 and, in revised form, July 11, 1995.

1991 Mathematics Subject Classification. Primary 20F18, 55P60, 55P62.

Research supported (in part) by a grant from the City University of New York PSC-CUNY Research Award Program.

${ }^{1} \mathrm{~A}$ proof in the case of finitely generated nilpotent groups can be given as follows: Since $L_{(0)} \cong M_{(0)}$, use [2, Theorem I.3.3] to find homomorphisms $\sigma: L \rightarrow Q, \tau: M \rightarrow Q$ into a third finitely generated nilpotent group $Q$ inducing rational isomorphisms. It follows that the kernels 
We noted in passing in [9, section 2] that one could even find rationally equivalent, 1-connected finite $\mathrm{CW}$-complexes such that there are no maps between $X$ and $Y$ in either direction inducing a rational equivalence $X_{(0)} \simeq Y_{(0)},{ }^{2}$ but the method outlined in [9] does not lead to an analogous example of finitely generated nilpotent groups. Our aim in this note is to modify the approach in [9] so as to yield:

Theorem. There exist finitely generated, torsion-free nilpotent groups $L$ and $M$ such that (i) $L_{(0)} \cong M_{(0)}$, (ii) there are no homomorphisms between $L$ and $M$ in either direction inducing a rational isomorphism.

This Theorem represents a sharpening of [7, Theorem 2.2]. Both theorems depend on the existence of J. Dyer's finite-dimensional, rational nilpotent Lie algebra $\mathcal{L}$, possessing the property that every automorphism of $\mathcal{L}$ induces the identity automorphism on the abelianization of $\mathcal{L}([1])$. The groups $L$ and $M$ are lattices in the rational nilpotent group corresponding to $\mathcal{L}$ under the classical log-exp equivalence, constructed in such a way as to have isomorphic $\ell$ - localizations for all but two primes $\ell$.

As a consequence of the Theorem, we infer:

Corollary. There exist nilpotent finite $C W$-complexes $X$ and $Y$ such that (i) $X_{(0)}$ $\simeq Y_{(0)}$, (ii) there are no maps between $X$ and $Y$ in either direction inducing a rational equivalence.

The spaces $X$ and $Y$ in the Corollary are not 1-connected, and to produce 1connected examples, as in [9], more work is needed. After proving the Theorem and Corollary in the next section, we make a comparison of the techniques leading to the Corollary and to the example in [9].

I would like to thank the referee for a number of helpful observations, in particular for supplying the Remark following the proof of the Theorem in section 2 .

\section{Proofs And COMmentary}

To prove the Theorem, we modify the procedure discussed in [7, section 2]. Following [7, section 2], start with a finite-dimensional, rational nilpotent Lie algebra $\mathcal{L}$ with the property that any Lie algebra automorphism of $\mathcal{L}$ induces the identity on $A b(\mathcal{L})$, the abelianization of $\mathcal{L}$, and let $N$ be the rational nilpotent group corresponding to $\mathcal{L}$ under the log-exp equivalence. Then take $H$ to be the subgroup of $N$ generated by a set $\left\{x_{1}, \ldots, x_{m}\right\}$ which maps onto a basis of $A b(N)$, the abelianization of $N$ (the latter being a rational vector space); $H$ is a finitely generated, torsion-free nilpotent group of class 6 .

Now consider a composite

$$
H \stackrel{\alpha}{\longrightarrow} \mathbb{Z}^{m} \stackrel{\beta}{\longrightarrow} \mathbb{Z} / p q
$$

$\alpha$ being the evident epimorphism and $\beta$ being an arbitrary epimorphism to the cyclic group of order $p . q$, where $p$ and $q$ are distinct primes. Define $K$ to be the Kernel of $\beta \circ \alpha$ and write $i: K \rightarrow H$ for the inclusion. Of course, $i$ is an $\ell-$ isomorphism for all primes $\ell \neq p, q$.

of $\sigma$ and $\tau$ have finite order and also that the indices $[Q: \sigma(L)]$ and $[Q: \tau(M)]$ are finite. Then $L_{(\ell)} \cong M_{(\ell)}$ for any prime $\ell$ not dividing the orders of the two kernels or the two indices.

${ }^{2}$ Unlike the situation in the infinite case, the spaces $X$ and $Y$ in the finite case cannot be taken to be rational $\mathrm{H}$-spaces or rational co-H-spaces; see [5, Corollary 1.4]. 
Next take a decomposition of the set of all primes into disjoint subsets $P, Q$ with $p \in P, q \in Q$ and construct the pullbacks ("Zabrodsky mixes")

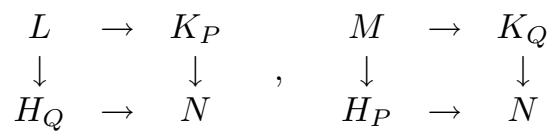

Here, $H_{P}$ and $K_{P}$ denote the $P$ - localizations of $H$ and $K$ and similarly for $H_{Q}$ and $K_{Q}$; moreover, the four homomorphisms $H_{P} \rightarrow N, H_{Q} \rightarrow N, K_{P} \rightarrow N, K_{Q} \rightarrow N$ in (2.1) are all rationalizations. It is immediate from (2.1) that

$$
L_{(0)} \cong N \cong M_{(0)} \text {; }
$$

furthermore,

$$
L_{P} \cong K_{P}, L_{Q} \cong H_{Q}, M_{P} \cong H_{P}, M_{Q} \cong K_{Q}
$$

If there were a homomorphism $\phi: L \rightarrow M$ inducing an isomorphism $\phi_{(0)}: L_{(0)} \cong$ $M_{(0)}$, then $\phi_{Q}: L_{Q} \rightarrow M_{Q}$ would likewise induce $\phi_{(0)}$. In view of $(2.2)$, we would thus have a homomorphism $\psi: H_{Q} \rightarrow K_{Q}$ inducing an isomorphism $\psi_{(0)}: H_{(0)} \stackrel{\cong}{\longrightarrow}$ $K_{(0)}$. Composing $\psi_{(0)}$ with the isomorphism $i_{(0)}: K_{(0)} \stackrel{\cong}{\longrightarrow} H_{(0)}$ would give an automorphism of $N$ which does not induce the identity on $A b(N)$, in violation of the manner in which $N$ was constructed. Similarly, any homomorphism $\phi^{\prime}: M \rightarrow$ $L$ inducing an isomorphism $\phi_{(0)}^{\prime}: M_{(0)} \stackrel{\cong}{\longrightarrow} L_{(0)}$ would yield a homomorphism $\phi_{P}^{\prime}: M_{P} \rightarrow L_{P}$ inducing $\phi_{(0)}^{\prime}$. Appealing once more to $(2.2)$, we would have a homomorphism $\psi^{\prime}: H_{P} \rightarrow K_{P}$ inducing an isomorphism $\psi_{(0)}^{\prime}: H_{(0)} \stackrel{\cong}{\longrightarrow} K_{(0)}$ and the composite $i_{(0)} \circ \psi_{(0)}^{\prime}$ would again lead to an illicit automorphism of $N$.

It remains to show that $L$ and $M$ are finitely generated (it being plain that they are torsion-free). To this end, it suffices to check that $A b(L)$ and $A b(M)$ are finitely generated; cf. [2, Proposition I.5.2] for a more general result. But

$$
\begin{aligned}
& A b(L)_{P} \cong A b\left(L_{P}\right) \cong A b\left(K_{P}\right) \cong A b(K)_{P}, \\
& A b(L)_{Q} \cong A b\left(L_{Q}\right) \cong A b\left(H_{Q}\right) \cong A b(H)_{Q} .
\end{aligned}
$$

Since $K$ and $H$ are finitely generated, it follows that $A b(L)_{P}$ is a finitely generated $\mathbb{Z}_{P}$ - module and that $A b(L)_{Q}$ is a finitely generated $\mathbb{Z}_{Q}$ - module. According to $[2$, Theorem I.3.10], $A b(L)$ is then a finitely generated $\mathbb{Z}$ - module. Similarly, $A b(M)$ is a finitely generated $\mathbb{Z}$ - module and the proof of the theorem is complete.

Remark. Fixing a prime $p$ and taking the kernels of a sequence of epimorphisms

$$
H \stackrel{\alpha}{\longrightarrow} \mathbb{Z}^{m} \longrightarrow \mathbb{Z} / p^{r}, r \geq 1,
$$

compatible with the natural projections $\mathbb{Z} / p^{r+1} \rightarrow \mathbb{Z} / p^{r}$, we obtain an infinite descending chain of subgroups of $H$, all rationalizing to $N$, but no two of which have isomorphic $p$ - localizations.

Proof of Corollary. Let $X$ and $Y$ be the Eilenberg-Mac Lane spaces $K(L, 1)$ and $K(M, 1)$. Statements (i) and (ii) of the Corollary are immediate consequences of the corresponding statements in the Theorem. Thus, it remains to establish that $X$ and $Y$ are (up to homotopy) finite $\mathrm{CW}$-complexes.

As $L$ is a finitely generated, torsion-free nilpotent group, it embeds as a discrete, uniform subgroup of a 1-connected nilpotent Lie group $\mathcal{M}(L)$ (Malcev). But $\mathcal{M}(L)$ 
is contractible and so the homogeneous space $\mathcal{M}(L) / L$, which is a closed, finitedimensional, smooth manifold, is a $K(L, 1)$. It follows, a fortiori, that $X$ has the homotopy type of a finite CW-complex and similarly for $Y$.

To obtain 1-connected finite CW-complexes (though not closed, smooth manifolds) satisfying the two statements of the Corollary, we could mimic our proof of the Theorem, relying however on the results of [7, section 3] (as corrected in the Erratum). ${ }^{3}$ As with the groups $L$ and $M$, the resulting spaces $X$ and $Y$ would be $\ell$ - equivalent for all but two primes. The approach outlined in [9, section 2] to obtain suitable spaces $X$ and $Y$ also used [7, section 3] but in a different way from that used here.

We first constructed a pair of 1-connected finite $\mathrm{CW}$-complexes $A$ and $B$ such that $A$ and $B$ are $\ell$ - equivalent for all but one prime $p$ and for which there exists a map $A \rightarrow B$ inducing a rational equivalence $A_{(0)} \stackrel{\simeq}{\longrightarrow} B_{(0)}$ but for which there is no such map $B \rightarrow A$ in the opposite direction. Then we constructed a second pair of 1-connected finite $\mathrm{CW}$-complexes $C$ and $D$ satisfying exactly the same conditions as the first pair except that the connectivities of $C$ and $D$ exceed the dimensions of $A$ and $B$. Finally, we took $X=A \vee D, Y=B \vee C$ and observed that $X$ and $Y$ are 1-connected finite $\mathrm{CW}$-complexes satisfying the two statements of the Corollary and such that $X$ and $Y$ are $\ell$ - equivalent for all $\ell \neq p$.

The approach just described exploits the "positively graded" character of 1connected spaces. The crucial property of being able to select a second pair $\{C, D\}$ which does not "interfere with" the first pair $\{A, B\}$ cannot, in any evident way, be replicated in nilpotent groups. Note also that we used that the coproduct in the homotopy category preserves 1-connectedness. On the other hand, the coproduct in the category of groups (free product) fails to preserve nilpotence; likewise, the coproduct in the homotopy category fails to preserve nilpotence. ${ }^{4}$ Thus, direct mimicking of the procedure in the previous paragraph to establish our Theorem is doomed to failure.

Nevertheless, it is legitimate to ask whether the Theorem (and the Corollary!) can be improved.

Question. Can $L$ and $M$ in the Theorem be chosen in the same Mislin genus, i.e. for every prime $\ell, L_{(\ell)} \cong M_{(\ell)}$ ?

In this connection, it is worth recalling that in [6, Example 3.11], Møller displayed examples of finite type, 1-connected, infinite $\mathrm{CW}$-complexes $X$ and $Y$, in the same Mislin genus, but such that there are no maps between $X$ and $Y$ in either direction inducing $X_{(0)} \simeq Y_{(0)}$; in this example, $X=B S^{3}$ but $Y$ is not a "familiar" space. A similar example, with $X=K(\mathbb{Z}, 2) \times \Omega S^{3}$, or even the $k$-fold iterated suspension of $K(\mathbb{Z}, 2) \times \Omega S^{3}, k \geq 1$, was discussed in [9, Example 2.3 (ii)].

\footnotetext{
${ }^{3}$ We use the word "mimic" rather loosely here. The rational Lie algebra theory appropriate to the study of 1-connected CW-complexes is an appropriate homotopy category of rational, reduced, free DGL's and is quite different from the classical category of (ungraded) rational, nilpotent Lie algebras.

${ }^{4}$ One might even conjecture that the coproduct $\mathcal{U} \vee V$ of two noncontractible nilpotent spaces is nilpotent $\Leftrightarrow \mathcal{U}$ and $V$ are 1-connected. However, the example $\mathbb{R} P^{\infty} \vee \Sigma \mathbb{R} P^{2}$ shows this to be false.
} 


\section{REFERENCES}

1. J.L. Dyer, A nilpotent Lie algebra with nilpotent automorphism group, Bull. Amer. Math. Soc. 76 (1970), 52-56. MR 40:2789

2. P. Hilton, G. Mislin and J. Roitberg, Localization of nilpotent groups and spaces, Notas de Matemática, North-Holland Mathematics Studies 15 (1975). MR 57:17635

3. C.A. McGibbon and J. Roitberg, Phantom maps and rational equivalences, Amer. J. Math. 116 (1994), 1365-1379. MR 95j:55026

4. C.A. McGibbon and J. Roitberg, Phantom maps and rational equivalences, II, Bol. Soc. Mat. Mex. 37 (1992), 367-381.

5. M. Mimura and H. Toda, On $p$-equivalences and $p$-universal spaces, Comment. Math. Helv. 46 (1971), 87-97. MR 44:2231

6. J. M. Møller, The normalizer of the Weyl group, Math. Ann. 294 (1992), 59-80. MR 94b:55010

7. J. Roitberg, Rational Lie algebras and p-isomorphisms of nilpotent groups and homotopy types, Comment. Math. Helv. 50 (1975), 1-8. Erratum, ibid. 51 (1976), 435-436. MR 51:1813; MR 54:13909

8. J. Roitberg, Computing homotopy classes of phantom maps, CRM Proceedings and Lecture Notes 6 (1994), 141-168. MR 95f:55010

9. J. Roitberg, The Mislin genus, phantom maps and classifying spaces, Topology and its Applications 69 (1996), 83-100.

Department of Mathematics \& Statistics, Hunter College (CUNy), 695 Park Ave., New York, New York 10021

Department of Mathematics, Graduate School (CUNY), 33 West 42 St., New York, NeW York 10036

E-mail address: jroitber@shiva.hunter.cuny.edu 